\title{
Trends and National Needs of China's Public Health
}

\subsection{Current Status and Characteristic on China's Public Health}

It is widely recognized among governments and public in the world that the public health has a direct influence upon economic development and social progress of countries, and it is an important base for constructing a harmonic society. Rapid development of science and technology has exerted unprecedented influences in the field of the public health, including the life span and the quality of life, the mechanism of the human body and combat against diseases. Current status and characteristic of the public health are described below.

\subsubsection{Population Pressure and Ageing Society}

According to UN statistical data, world population will continue to grow to 9 billion by 2050, with the most of the increase in underdeveloped countries. Thus, in the coming 40-50 years, the proportion of child-bearing women will reach $40 \%$ of the total population. By 2030, the young population aged 10-19 is estimated to grow to 1.3 billion. Strict birth-control policy adopted in China more than 30 years has effectively curbed its excessive population growth. However, the population pressure still remains high due to a large population base. It is estimated that by the fifties of this century, the population peak in China will appear at around 1.5 billion.

For nearly two hundred years, most of the developed countries have witnessed a continuous increase in the age of population ${ }^{[1]}$, and the mankind moves into an ageing society. Along with rapid development of the socioeconomy in China, the population age has also increased remarkably. At present, we have 143 million people aged above 60. As estimated, late forties of this century will see a peak in the ageing population: people aged above 60 will reach 430 million, making up $30 \%$ of the total population ${ }^{[2]}$. 


\subsubsection{Reproductive Health Faces Challenges}

Reproductive health has become an important global issue in the field of the public health. At the 2005 World Summit Meeting and the $60^{\text {th }} \mathrm{UN}$ General Assembly, it was agreed that the goals of promoting reproductive health should be included in the UN millennial objective. The Chinese government also attaches great importance to the research on population control and reproductive health. In the "Outline of China's mid and long-term scientific and technological development plan", the safe contraception and birth-defect prevention are listed as the first of five priority subjects in the field of the public health.

The contraception measures in the world have been adopted at $64 \%$ by 2005, but it was not evenly distributed, and about 400 million married women did not take any contraceptive measure. On the other hand, there exists a serious problem of continuously increasing infertility rate in the world. Such couples make up 15\% in developed countries, and even approach to $30 \%$ in a number of European countries. In China, such population has also increased greatly, from less than $3 \%$ in the seventies of last century to $8-10 \%$ at present.

Birth defects include congenital malformation as well as congenital mental retardation and inherited metabolic disease. At present, the birth-defect rate in China is as high as $5 \%$, thus causing a heavy burden to the society and families. Birth defect has become one of the main causes for infant mortality and children/adult disability in China.

\subsubsection{Nutrition-related Health and Food Safety Issues Becoming Increasingly Important}

Food nutrition is a vital issue for the people's livelihood and their descendents' health. Maintaining a healthy diet and lifestyle is internationally recognized as an effective strategy and economical policy for preventing chronic diseases such as cardiovascular disease, diabetes type II and malignant tumors. Chinese government has always attached great importance to the improvement of nutrition and health for the Chinese people, and in 1992, it signed the "World's declaration of nutrition" and "World's nutritive action plan". The importance of these reforms recently promulgated by the State Council emphasizes the subject of "advocating healthy and civilized lifestyle and promoting properly nutrition for the public".

Like many other developing countries, China now faced the challenges of both excessive and deficient nutrition. Within dozens of years, China has been transformed and adapted a style of Western-like high fat diet, which took 200 years for Western countries to adapt. Excessive eating and reduction of physical activities is now the main reason for rapid prevalence of obesity, hypertension, type II diabetes, cardiovascular disease and some tumors ${ }^{[3,4]}$. On the other hand, malnutrition remains to be a major problem of health in the poverty-stricken areas.

Food safety in China becomes a serious problem, since the country 
faces the food contamination by microbes or residual pesticides, as well as environmental pollutions. In the meantime, anti-nutritional factors, transgenic food and food supplements lack full safety assessment. All of these problems constitute the major challenges to the food safety.

\subsubsection{Disease Spectrum has been Changed}

Thanks to social progress, scientific and technological development, the scope and degree of harmful to people's health caused by infectious diseases or puerperal and perinatal diseases have decreased considerably, which are the major threats to human being in the early stages of civilization. While the chronic diseases such as cancer, cardiovascular, metabolic and neurological diseases have become the major threats to mankind. Based on a report on the chronic diseases in 2006, the World Health Organization pointed out that the number of people who die of chronic diseases already made up 60\% of the total number of deaths. To make the matters worse, this figure is set to increase rapidly, by about $17 \%$ in the coming 10 years ${ }^{[5]}$. Chronic diseases have become the major causes of death for both urban and rural inhabitants. In many poverty-stricken counties in China, the death resulted from the chronic diseases reaches as high as $60 \%{ }^{[6]}$.

Chronic diseases are mostly life-long diseases with bad prognosis, often accompanied by severe complications, thus greatly decreasing the quality of life of inflicted patients. Therefore, the chronic diseases significantly affect the health of the working population in China. For example, about half of patients seeing doctors in 2003 belonged to malignant tumors, cerebrovascular related diseases, heart disease, hypertension and diabetes ${ }^{[6]}$.

Ageing population leads to a considerable increase of neurodegenerative diseases, which is now the fourth threat of older people's health after cardiovascular/cerebrovascular disease, cancer and stroke. In the meanwhile, due to rapid economic development, accelerated social tempo, sharpened social competition, the increase of pressure for work and study, as well as changes in spiritual activities, morbidity of mental diseases has gone up remarkably. Therefore, the mental diseases now stand as the number one in terms of total disease-burden, making up one fifth of the total burden of all kinds of diseases.

We are now in the age of information with internet, wireless communication and other modern information-technologies. Internet related behaviors become an important part of people's daily life. The number of people using the internet in China have been reached 298 millions ${ }^{[7]}$.These kinds of new behavior changes in the information society result in the new cognitive or mental problems such as the internet addiction. It was reported that the number of internet-addiction people in China in 2007 reached 8 millions.

\subsubsection{Infectious Diseases Remain a Serious Challenge}

Despite of the great success in the battle against infectious diseases, the globalization, the changes in the ecological environment and economic 
development, as well as terrorism and other social unstable factors, researchers as well as the whole society have always paid great attention to the harm of emerging and recurrent infectious disease. The outburst of SARS caused by a new coronavirus in China and other countries in 2003 resulted in tremendous economic losses and social negative effect to many countries, while in recent years the whole world has been highly alert on the potential danger of prevalence of the avian and human influenza.

Despite the great progress achieved in understanding and treating infectious diseases such as hepatitis and AIDS, no highly effective therapies have been developed. Since these viruses have strong genetic variability, their infection mechanism is still not well understood. The occurrence of tubercle bacillus that is capable of resisting various antibiotics has lead to a new outbreak of tuberculosis. Since there is lack of effective therapies for treating tuberculosis, prevention and control of these infectious diseases pose a new challenge to us.

\subsection{Influence of the Modern Science and Technology and the Trends on Public Health}

Life science has been rapid developed and made great influence in the scope of natural sciences since 1950's. In the coming 20-30 years, mankind will achieve major progress in the understanding of the origin and evolution of life, structural and functional development of the brain and nerves. The progress regarding the subjects of genomics, proteomics and bioinformatics will enable scientists to have a better understanding of inheritance, growing and ageing, metabolism, immunity, ecology, evolution as well as biodiversity systematically on the molecular level, thus linking and integrating macro- and micro-biology.

\subsubsection{Impact of Modern Science and Technology on Public Health}

The progress made in the field of life science has lead to a better understanding of health and diseases. Modern biological disciplines such as molecular biology, cell biology, genetics and immunology, has integrated with clinical research and created new disciplines, including medical genetics, biomedical engineering and immunological therapeutics. Therefore, National Institutes of Health in the US has located molecular biology, cell biology, genetics, immunology and other biological disciplines into the category of biomedicine so that basic research of life science and medical research could be more closely linked and uniformly supported.

The quick development of the life science in the $20^{\text {th }}$ century has benefited from the integration of different fields such as physics, chemistry and mathematics. Through physical and chemical means, scientists have established modern molecular biology, discovered that DNA is a genetic material, established the principles of gene expression and regulation, and developed 
technologies for making recombinant DNA, thus laying a solid foundation for further research in the entire field of public health. Advancement in DNA sequencing techniques led to the successful sequencing of the whole human genome, which can now be used to understand the genetic basis of diseases and will undoubtedly lead to the development of new medicines that will benefit the population at large.

Through successful application of technologies in the field of public health such as molecular imaging technology, scientists have brought about a revolution in the field for perception of the human body. The contribution of chemical technology is seen in the rapid development in the field of chemical biology for understanding of signal transduction pathways, as well as the regulation of living processes by small molecular compounds. The invention and maturity of micro detection technique discloses the life complexity. The combination of medicinal chemistry and genomics is now giving rise to new concepts and platform technologies for development of new drugs.

\subsubsection{Trends of Science and Technology on Public Health}

In the last half century, science and technology has played a very important role in respect to prevention, diagnosis, therapy and rehabilitation of diseases, and promoted the modernization of medicine, thus generating two major high-tech pillar industries of the modern medical system - biomedical engineering and pharmaceutical industry. On the one hand, demands in the public health give a great impetus for the development and application of new technologies. On the other hand, breakthroughs in new technologies provide good solutions for the progress in the field of the public health. Focusing on the development of science and technology should be a priority for the public health.

The first emphasis should be on the development and improvement of research technologies of life science based on physics and chemistry, including synchronous radiation and nuclear magnetic resonance technologies, and cryo-electron microscopy for measuring the structures of macromolecules; the monomolecular measurement and manipulation technology; synthetic biological technology for synthesizing genes through micro fluid chip; highthroughput and low-cost genomic sequencing technology; the genetic manipulation technology as well as the molecular markers in vivo and molecular imaging technology. Furthermore, the involvement of information technology helps to greatly improve the data acquisition and processing ability in life science; and automatic-analysis technology and mathematical models will change the means and methods of life science in a revolutionary way.

A new frontier in life science is stem cell research, which will not only integrate the progress already achieved in the other major disciplines in the field of life science, but also create new model and means for disease diagnosis and therapy - a major issue in the field of the public health. The field of stem cell research will provide a new opportunity for the improvements on the public 
health.

Another important growing area in the future for life science research is in the field of brain science, and its key indication lies in the transformation from human exploration of the external world to its exploration of human itself, from exploration of material world to exploration of the spiritual world. Through generations of technical improvement, brain science can now give answers to a considerable number of questions regarding consciousness, but there still remains much to learn about the human mind/brain.

In recent years, there are rapid progress in international development of drug innovation. First, new disciplines of life science, such as genomics, proteomics, biological chip, transgenic animal and biological informatics, have been combined with pharmaceutical research. The aim is now finding and verifying new drug-targets and studying the efficacy of drugs. Second, emerging new scientific disciplines are quickly integrated into the drug discovery process.

\subsubsection{Transformation of Biomedicine}

Along with an increasingly closer connection between modern biology and clinical medicine, basic research, applied research and industrial R\&D are combined at high speed. In the future, basic biological research and clinical application research will be more closely related, and research results will be industrialized at a more rapid speed. In the past, a successful translation from a new discovery in a laboratory (publication of articles or patents) to clinical application needs $4-50$ years, averaging 17 years ${ }^{[8]}$. To strengthen the marriage between clinical research and basic research, the USA and UK, the countries with the fastest development in life science and medical science, present a new development model for biomedicine called translational research, characterized by integration of basic biological research and clinical application where clinical demands always play an important part from the beginning of research as well as all later subsequent stages.

As life is a sophisticated networked system, exploring the essence of life and disease from the perspective of a system has become a new international frontier in the field of life science. In recent years, the rapid development of systems biology, a newly emerging subject on complicated biological systemsprovides new ideas and methods for understanding/treating complicated diseases like tumor and diabetes. Systems biology is consistent with the general ideas of traditional Chinese medicine, thus the development of the systems biology in China provides an opportunity for the modernization of traditional Chinese medicine.

\subsection{The Needs of China's Public Health}

Based on the major trends in the field of the public health as well as the progress achieved in life science, the following strategic needs will be taken into 
account in the field of the public health in China.

\subsubsection{The Needs on Improving China's Innovative Ability in Biomedicine}

Although the life science in China has developed remarkably and the ranking in the clinical medical research in China has risen from the $23^{\text {rd }}$ in 2003 to $17^{\text {th }}$ in 2005 , there is still a big gap when compared with most developed countries. Therefore, the support for life science in China must be strengthened in order to rapidly improve the innovative ability in biomedicine. Furthermore, the future progress in life science will encourage multidisciplinary research between biologists and those scientists working on other subjects such as mathematics, physics and chemistry, since life science is now at a new stage where inter-disciplinary support is needed for its further development.

The key points for improving China's innovative ability in biomedicine are to strengthen the basic research in life science. The field of the public health should prioritize the basic researches in molecular biology, cell biology and developmental biology and so on. Attention should be given to understand the relationship between epigenetic regulation and the diseases, the structures and functions of non-coding RNA, as well as the self-renewal and directional differentiation of stem cells. Moreover, attention should be paid to the development of newly emerging fields such as systems biology and synthetic biology so as to make major breakthroughs in the frontier areas of life science.

\subsubsection{The Needs on Controlling Population and Improving Birth Quality}

As a country with the largest population, China implements its basic national policy on birth control. Present contraception methods alone, is not adequate to meet the requirements of reproductive health. It is necessary to strengthen basic research in reproductive medicine and develop a new generation of safe, effective and convenient contraception methods. Furthermore, we have to take into account the individual differences when responding to contraceptives. Different cultures and economic backgrounds also determine the choices of fertility regulation.

While controlling the number of births, we need to improve the quality of reproductive health. Intensified researches and key techniques in the field of reproduction and developmental biology will help reduce the number of defects in newborns and improve the level of prepotency. Furthermore, in view of the continual increase in the infertility rate in the world, we have to strengthen auxiliary reproductive technologies to prevent and cure infertility.

\subsubsection{The Needs on Public Health and Physical-mental Health}

Major chronic diseases such as cancer, cardiovascular, cerebrovascular, metabolic and neurological diseases have become the major menaces for the Chinese population. Along with the socio-economic development in China and 
the increase of the ageing population, the threats of chronic diseases must be significantly increased. The strategic needs in the public health is to strengthen prevention and control of these chronic diseases, focus on preventive measures, and push ahead with the strategic transformation of medical model from past reliance on disease treatment to reliance on prevention, prediction and intervention.

Along with tremendous changes in social culture such as rapid the development of science and technology, neurological and mental diseases represented by depression and neuro-degenerative diseases become an epidemic disease of the $21^{\text {st }}$ century. It has become an imperative demand of the nation to study the fundamental course of the brain and acts, determine the genetic and environmental factors related to behavior and mental disorder, solve the grave social problem of cognitive, behavior and mental disorder and realize individual physical and mental health.

Food is the physical base on which human existence relies; it is also the physical base for the health of the human body. As a developing country, China is still faced with dual challenge of nutrient imbalance and deficiency. Therefore, we have to rapidly improve the nutritious research so that the nutrition science can satisfy the requirements on the public health and disease prevention, and provide the nutrient health of the people.

\subsubsection{The Needs on Guaranteeing Biological and Food Safety}

Along with global economic integration and rapid social development, the growth of internal and external population flow to and from various countries is becoming a major characteristic of contemporary society. Furthermore, struggle against international terrorism is now a serious issue. All these factors make biological safety an important part of national security. There is a need to establish and improve current contingency plans for public health in the event of a terrorist attack. The country needs to improve the quality of surveillance equipments and develop a network for biological safety, and raise our capacity for dealing with public security crises.

After promulgation of the "Food hygiene law of the People's Republic of China", the Chinese government implements food hygiene supervision regulations, thus integrating food safety into legal management. However, the food safety in China is faced with severe problems. At present, food-borne diseases is the primary factor affecting public health, and the potential menace for new biological and chemical pollutants in the food toward health is a new problem that should not be ignored. Furthermore, applications of new food technologies post a new challenge to food safety. Therefore, it is imperative to strengthen food safety research.

\subsubsection{The Needs on Developing Modern Medical Industry}

The stimulation of socio-economic development in life science is largely seen in the medical industry. At present, about $60 \%$ of all biological 
researches are in the area of medical sciences for the development of new drugs or improvement of conventional medicines. The total output of the medical industry in China makes up only around $2 \%$ of the total output of the country, and around $7 \%$ of the total output of the medical industry of the whole world. It is important for the medical industry in China to develop a number of "blockbuster" drugs in the forthcoming period.

The biomedical engineering industry is currently centralizing and integrating life science, physics, chemistry and engineering technology. The biomedical engineering industry and the pharmaceutical industry constitute two major parts of the modern medical industrial system. The industries stimulated by them are becoming more and more important in the national industrial economy. However, compared with developed countries, the medical apparatus enterprises in China are confined to a small scale, singular product with low technological level, and their international market shares are below $2 \%$. Therefore, it is an urgent issue that the medical apparatus industry improves its R\&D ability, upgrades the old products and raises the technological level of the products in the forthcoming period.

Traditional Chinese medicine has its own characteristics and advantages in the research field of the public health in China, and plays an important role in the respect therapy of complex chronic diseases as well as medical services in the rural areas. We should study sub-health and complex chronic diseases by using modern biological means as well as the traditional Chinese medicine characterized with integrity and systematization, so that the convergence of Eastern and Western medicines may be realized step by step in the near future. 